\title{
O ENFERMEIRO NA EDUCAÇÃO DE CUIDADORES DE PACIENTES COM SEQUELAS DE ACIDENTE VASCULAR CEREBRAL
}

\author{
NURSES' ROLE IN THE EDUCATION OF CAREGIVERS OF PATIENTS WITH STROKE
}

\author{
Renata Carmel de Araujo Silva ${ }^{a^{*}}$, Geyciele Lima Monteiro ${ }^{b^{*}}$, Ariane Gomes dos Santos ${ }^{c^{*}}$ \\ arennatacarmell@hotmail.com, bgeycielelima@hotmail.com, carianeg.santos@hotmail.com \\ *Instituto de Ensino Superior Múltiplo - Timon (MA), Brasil
}

Data de recebimento do artigo: 14/12/2014

Data de aceite do artigo: 04/05/2015

\section{RESUMO}

Introdução: Acidente vascular cerebral (AVC) é uma lesão súbita da função cerebral ocasionada pela deficiência de suprimento sanguíneo para o cérebro, é uma das maiores causas de morte e sequelas no mundo e no Brasil. Objetivos: Identificar a produção científica sobre a importância do enfermeiro na orientação aos cuidadores de clientes com sequelas de AVC; identificar as dificuldades enfrentadas pelos cuidadores no sistema de cuidado domiciliar e analisar a efetividade da assistência educacional para a clientela do estudo. Materiais e métodos: Trata-se de uma revisão integrativa da literatura que reuniu artigos das bases de dados LILACS e SciELO em português e espanhol, utilizando os descritores "acidente vascular cerebral and assistência domiciliar and cuidadores and enfermagem". A amostra final constituiu-se de 13 artigos. Resultados: Constatou-se que as dificuldades enfrentadas pelos cuidadores estão relacionadas à falta de informaçóes e orientaçôes sobre a patologia, suas complicaçôes e principais cuidados que precisam ser prestados no domicílio sendo o enfermeiro um profissional habilitado para fazer essa educaçáo em saúde, e que programas de alta hospitalar proporcionam ao cuidador mais segurança, confiança, permitindo melhorar a qualidade de vida tanto do paciente quanto do cuidador. Consideraçóes finais: É importante oferecer educação em saúde de qualidade aos pacientes e cuidadores durante o período de internação. $\mathrm{O}$ enfermeiro deve utilizar suas habilidades de educador como estratégia de cuidado permanente ao paciente após a alta hospitalar, por meio da minimização das dúvidas, medos e dificuldades dos cuidadores e da capacitação dos mesmos para enfrentar a nova realidade.

Palavras-chaves: Acidente vascular cerebral; assistência domiciliar; cuidadores; enfermagem.

\section{ABSTRACT}

Introduction: A cerebrovascular accident (CVA) is a sudden injury of brain function caused by blood supply deficiency in the brain, which has become a major cause of death and sequelae in the world as well as in Brazil. Objectives: To identify the scientific literature on the importance of nurse in the orientation of caregivers of customers with CVA sequelae; to identify the difficulties faced by caregivers at the home nursing system and to analyze the effectiveness of educational assistance to the clientele of the study. Methods: This is an integrative literature review that analyzed databases of articles LILACS and SciELO in Portuguese and Spanish, using the descriptors "acidente vascular cerebral and assistência domiciliar and cuidadores and enfermagem" ["stroke and home care and caregivers and nursing"]. The final sample consisted of 13 articles. Results: It was found that the difficulties faced by caregivers are related to lack of information and guidance on the pathology, its complications and the main cares that must be provided at home, the nurses being the professionals qualified to make this health education, and that the discharge programs provide the caregiver with more security, trust, thereby allowing to improve the quality of life of the patient and the caregiver alike. Final thoughts: It is important to offer an education on health quality to patients and caregivers during hospitalization. Nurses should use their skills as educators as a permanent care strategy to the patient after discharge, by minimizing the doubts, fears and difficulties of caregivers and empowering them to face the new reality.

Keywords: Stroke; home nursing; caregivers; nursing. 


\section{Introdução}

O acidente vascular cerebral (AVC) é uma lesão súbita da função cerebral ocasionada pela deficiência de suprimento sanguíneo para uma determinada área do cérebro, por oclusão de vasos gerando uma isquemia ou por uma ruptura dos vasos sanguíneos, ocorrendo uma hemorragia, dessa forma dividido em duas categorias: acidente vascular cerebral isquêmico (AVCI) e acidente vascular cerebral hemorrágico $(\mathrm{AVCH})$, respectivamente ${ }^{1}$.

O AVC se tornou uma das maiores causas de morte e sequelas no Brasil e no mundo, atingindo 16 milhões de pessoas a cada ano ${ }^{2}$. No Brasil, em 2011, foram registradas 179 mil internaçôes por AVC (isquêmico e hemorrágico $)^{3}$.

As incapacidades e sequelas manifestadas após um AVC envolvem o comprometimento motor de um lado do corpo, dificuldades na comunicação devido à dificuldade de articulação de palavras ou até mesmo à afasia, déficit visual e auditivo, sintomas de depressão ${ }^{4}$, comprometimento da deambulação do tônus e trofismo, dor articular ou muscular e déficit de coordenação 5

A pessoa acometida por um AVC se depara com a necessidade de mudar seus hábitos e estilo de vida, podendo comprometer a família, primeiro grupo de relaçóes em que o sujeito está inserido. Aparecem dúvidas quanto às causas e consequências, duração das sequelas, possibilidade de recuperação e principalmente como se comportar e agir diante dessa nova realidade. Diante disso, é necessário considerar que a família sofrerá um impacto significativo diante do evento mencionado. A falta de informações e orientações fornecidas por parte dos profissionais de saúde está associada às dificuldades encontradas após o $\mathrm{AVC}^{6}$.

A educação em saúde é uma ferramenta que visa melhorar a qualidade do cuidado. Todavia, para que isso aconteça, é necessário que as intervençóes educacionais sejam capazes de motivar o diálogo, a indagação, a reflexão e a ação partilhada, de forma que as orientaçóes e informaçōes transmitidas aos cuidadores possibilitem melhores cuidados às pessoas que deles necessitam. Cuidadores carecem de uma comunicaçáo mais eficaz e efetiva com os profissionais de saúde, que muitas vezes deixam o cuidador desamparado, sem compreender os cuidados específicos que necessitam ser prestados e como devem ser realizados, tornando assim o cuidado uma tarefa ainda mais difícil ${ }^{7}$.

O enfermeiro é um dos profissionais mais habilitados para fazer a educação em saúde pelo caráter holístico de sua formação e pelo fato deste passar mais tempo na assistência aos pacientes. No entanto, precisa estar consciente de que a internação é temporária e que a família deve estar capacitada para assistir os pacientes de maneira permanente, necessitando, dessa forma, de orientaçáo para o cuidado em domicílio ${ }^{8}$.

Sendo assim, a família do indivíduo com sequelas do AVC é o centro da prestação de cuidados no domicílio. Dessa forma, deve ser alvo da assistência de enfermagem, pois o impacto gerado pela doença pode ocasionar dificuldades para o cuidado com o ente. Assim, o enfermeiro deve estabelecer uma relação de confiança e de parceria com os familiares para que estes tenham maior habilidade durante o cuidado com o paciente que perdeu a capacidade de autocuidado total ou parcialmente por causa de sequelas do $\mathrm{AVC}^{6}$.

A realizaçáo desse estudo faz-se relevante pelo fato do AVC ser uma doença de elevada incidência e por possuir grande potencial para causar incapacidades que podem afetar a qualidade de vida do indivíduo e da família.

Diante do exposto, faz-se necessário analisar a importância de intervençóes educacionais em saúde para transmitir aos cuidadores de clientes com as sequelas do AVC, informaçóes e orientaçóes sobre as necessidades de cuidados que precisam ser prestados e que podem resultar em um cuidado mais efetivo, proporcionando melhor qualidade de vida para esses indivíduos e suas famílias.

Os objetivos do presente estudo foram: identificar a produção científica sobre a importância do enfermeiro na orientaçáo aos cuidadores de clientes com sequelas de AVC; identificar as dificuldades enfrentadas pelos cuidadores no sistema de cuidado domiciliar; analisar a efetividade da assistência educacional para a clientela do estudo.

\section{Materiais e métodos}

Trata-se de uma revisão integrativa da literatura. Para a elaboração dessa revisão integrativa, as seguintes etapas foram percorridas: identificação do tema e seleçáo da questão norteadora da pesquisa, amostragem, categorização dos estudos, avaliação dos estudos, interpretação dos resultados e síntese do conhecimento.

Esse estudo foi guiado pela seguinte questão norteadora: qual a importância da assistência educacional feita por enfermeiros aos cuidadores de pacientes sequelados após acidente vascular cerebral?

A coleta de dados foi realizada no período de fevereiro a junho de 2014. Para a seleçáo dos artigos utilizou-se a base de dados LILACS (Literatura LatinoAmericana e do caribe em Ciências de Saúde) e SciELO (Scientific Eletronic Library online) com o recorte temporal de 1999 a 2014. Os descritores extraídos do DeCS (Descritores em Ciências da Saúde) do Portal BVS (Biblioteca Virtual em Saúde) Bireme (Biblioteca Regional de Medicina) foram: "acidente vascular 
cerebral and assistência domiciliar and cuidadores and enfermagem".

Os critérios de inclusão estabelecidos para a revisão foram: artigos completos disponíveis eletronicamente; publicados nos idiomas português e espanhol no período de 1999 a junho de 2014; retratando a assistência educacional feita por enfermeiros aos cuidadores de pacientes vítimas de sequelas após acidente vascular cerebral que responderam a questáo norteadora deste estudo. $\mathrm{O}$ critério de exclusão estabelecido foi: artigos não disponíveis na íntegra.

Foram encontrados 100 artigos; após aplicação dos critérios de inclusão e exclusão, selecionou-se 13 artigos com relevância para o estudo, constituindo-se assim a amostra final.

Para a coleta de dados foi utilizado um instrumento elaborado pelas pesquisadoras, que contempla as seguintes variáveis: ano de publicação, título, objetivo, características metodológicas do estudo, principais resultados encontrados e a conclusão de cada estudo.

Os resultados foram analisados de forma descritiva tendo por base a literatura científica sobre a temática em questáo.

\section{Resultados}

Foram analisados 13 artigos na íntegra, e após elaboração do Quadro 1, que apresenta a caracterização geral deles, observou-se o predomínio de pesquisas no ano de 2009, 4 artigos (30,9\%). Prevaleceram publicaçóes no idioma português, totalizando 12 artigos $(92,3 \%)$; e com abordagem qualitativa, 7 artigos $(53,8 \%)$ (Quadro 1$)$.

Quadro 1: Caracterização dos artigos selecionados de acordo com título, autores, ano de publicação e desenho de estudo.

\begin{tabular}{|c|c|c|c|}
\hline Titulo & Autores & Ano de publicaçáo & Abordagem \\
\hline $\begin{array}{l}\text { Sobrecarga dos cuidadores de idosos com acidente vascular } \\
\text { cerebral }\end{array}$ & $\begin{array}{c}\text { Pereira RA, Santos EB, } \\
\text { Fhon JRS, Marques S, } \\
\text { Rodrigues RAP }\end{array}$ & 2013. & Quantitativa \\
\hline $\begin{array}{l}\text { Adaptação da pessoa após acidente vascular encefálico e seu cui- } \\
\text { dador: ambiente domiciliar, cadeira de rodas e de banho }\end{array}$ & $\begin{array}{c}\text { Garanhani MR, Alves JF, } \\
\text { Fugisawa DS, Garanhani } \\
\text { ML }\end{array}$ & 2010. & Qualitativa \\
\hline $\begin{array}{c}\text { Calidad de vida de cuidadores de adultos com accidente } \\
\text { cerebrovascular }\end{array}$ & $\begin{array}{l}\text { Torres IEF, Prieto AM, } \\
\text { Lián AH }\end{array}$ & 2010 & Quantitativa \\
\hline $\begin{array}{l}\text { A experiência de pacientes e cuidadores após acidente vascular } \\
\text { encefálico: uma revisão narrativa da literatura }\end{array}$ & $\begin{array}{l}\text { Garanhani MR, Ferreira } \\
\text { AMDM, Silva CK, } \\
\text { Laskovsk L, Moreira MD, } \\
\text { Garanhani ML. }\end{array}$ & 2009. & Qualitativa \\
\hline $\begin{array}{l}\text { A problemática do cuidador familiar do portador de acidente } \\
\text { vascular cerebral }\end{array}$ & $\begin{array}{l}\text { Andrade OG, Rodrigues } \\
\text { R A P. }\end{array}$ & 2009. & Quantitativa \\
\hline $\begin{array}{l}\text { A vivência do cuidador familiar de vítima de Acidente Vascular } \\
\text { Encefálico: uma abordagem interacionista }\end{array}$ & $\begin{array}{l}\text { Machado ALG, Jorge } \\
\text { MSB, Freitas CHA. }\end{array}$ & 2009. & Qualitativa \\
\hline $\begin{array}{l}\text { O cuidado domiciliar de idosos acometidos por acidente vascular } \\
\text { cerebral: cuidadores familiares }\end{array}$ & $\begin{array}{c}\text { Souza CB, Abreu RNDC, } \\
\text { Brit EM, Moreira TMM, } \\
\text { Silva LMS, Vasconcelos } \\
\text { SMM. }\end{array}$ & 2009. & Quantitativa \\
\hline $\begin{array}{l}\text { Cuidar de pessoa incapacitada por acidente vascular cerebral no } \\
\text { domicílio: o fazer do cuidador familiar }\end{array}$ & $\begin{array}{l}\text { Perlini NMOG, Faro } \\
\text { ACM. }\end{array}$ & 2005. & Quantitativa \\
\hline $\begin{array}{l}\text { Interação cuidador familiar-pessoa com AVC: autonomia } \\
\text { compartilhada }\end{array}$ & Bocchi SCM, Angelo M. & 2005. & Qualitativa \\
\hline $\begin{array}{l}\text { Percepçáo de cuidadores familiares sobre um programa de alta } \\
\text { hospitalar }\end{array}$ & Cesar AM, Santos BRL. & 2005. & Qualitativa \\
\hline $\begin{array}{l}\text { Educação em saúde e família: o cuidado ao paciente, vítima de } \\
\text { AVC }\end{array}$ & $\begin{array}{c}\text { Chagas NR, Monteiro } \\
\text { ARM } \\
\end{array}$ & 2004 & Qualitativa \\
\hline $\begin{array}{c}\text { O papel do enfermeiro como educador junto a cuidadores } \\
\text { familiares de pessoas com AVC }\end{array}$ & Bocchi SCM. & 2004. & Qualitativa \\
\hline $\begin{array}{l}\text { Abordagem Holística do sistema de cuidado familiar do idoso } \\
\text { com acidente vascular cerebral }\end{array}$ & $\begin{array}{l}\text { Andrade OG, Rodrigues } \\
\text { R A P. }\end{array}$ & 2002. & Qualitativa \\
\hline
\end{tabular}


Os 13 artigos selecionados foram comparados e agrupados por similaridade de conteúdos sob forma de categorias, sendo divididos em 3 grupos de análise: "dificuldades enfrentadas pelos cuidadores familiares", "enfermeiro como educador no sistema de cuidado domiciliar" e "preparo do cuidador para alta hospitalar".

\section{Dificuldades enfrentadas pelos cuidadores familiares}

As dificuldades enfrentadas pelos cuidadores familiares estâo relacionadas à falta de informaçôes sobre a doença e suas complicações, principais cuidados a serem realizados no domicílio, dessa forma gerando nos cuidadores insegurança e despreparo em exercer sua função de forma correta, comprometendo a saúde do paciente e de seu cuidador ${ }^{9,10,11,12,13}$.

Surgiram dúvidas a cerca da assistência que deve ser prestada no domicílio após a alta hospitalar relacionadas à alimentação, administração de medicamentos, complicaçôes clínicas, higiene corporal e oral, eliminaçóes, risco de acidentes e movimentação do paciente. Tais dúvidas são atribuídas ao fato do cuidador não receber orientaçóes básicas para dar continuidade ao tratamento no domicílio, gerando incapacidade e inquietaçáo sobre como cuidar ${ }^{10,11}$.

Surgiram também dificuldades com os cuidados que requerem força física como técnicas de transferência ou mobilização?. A dependência funcional, motora e cognitiva de pacientes de AVC caracteriza-se como uma dificuldade enfrentada pelos cuidadores devido à sobrecarga ocasionada ${ }^{14}$. Essa sobrecarga do cuidador gera o comprometimento da qualidade de vida do paciente e da família ${ }^{9}$, pois terão a saúde física e psicológica expostas aos efeitos de assumir esse papel, podendo comprometer o processo de reabilitaçáo do paciente e até provocar açốes extremas de submissão do doente a maus tratos $^{15}$

Portanto, oferecer orientações e informaçóes aos cuidadores de como lidar com os pacientes sequelados após um AVC no domicílio, capacitando-os para exercer sua função, minimiza a sobrecarga dos mesmos, proporcionando uma melhor qualidade de vida para ambos.

\section{Enfermeiro como educador no sistema de cuidado domiciliar}

Pelo desconhecimento sobre o cuidar de uma pessoa com AVC o cuidador familiar passa a lidar com situações em uma área que não tem domínio, e consequentemente se depara com a incerteza, o medo, a insatisfação e os perigos de cuidar do doente ${ }^{15,16}$. Para superá-los, o cuidador deve utilizar os conhecimentos adquiridos por meio das orientações de enfermagem, o que irá lhe conferir segurança para lidar com situaçóes relacionadas ao seu processo de cuidar ${ }^{15}$. O cuidador é uma importante fonte de cuidado informal, requerendo assim, apoio dos sistemas de saúde e dos profissionais de enfermagem ${ }^{17}$.

No papel de educador em saúde, o enfermeiro deve orientar os cuidadores, capacitando as famílias para o cuidado no domicílio, transformando a ansiedade e o despreparo destes em força produtiva orientada para o cuidado, cabendo-lhe por direito e dever cumprir esse papel $^{11}$.

Em pesquisa realizada com 35 cuidadores familiares principais de pessoas incapacitadas por AVC, constatou-se que para assegurar a garantia da continuidade do cuidado no domicílio, os cuidadores necessitam iniciar, ainda durante a hospitalização, o desenvolvimento da capacidade para cuidar, o que significa a realização de um preparo efetivo, no qual o enfermeiro esclareça as possíveis dependências do paciente e os cuidados iniciais que a família terá que tomar, assumindo com ela uma relaçấo de cooperação e apoio, devendo estar preparado para realizar esses cuidados e estimular o paciente e cuidadores a realizá-los de forma correta ${ }^{10}$.

Para isso, a enfermagem necessita valer-se de instrumentos que facilitem o processo de adaptação da família à nova situação, destacando-se, dessa forma, a criatividade e a sensibilidade do profissional diretamente com a equipe de saúde para possibilitar a continuidade dos cuidados no domicílio ${ }^{18}$.

Em um estudo realizado em um Centro de Saúde da Família situado em Fortaleza-CE, as cuidadoras familiares pesquisadas não mencionaram o enfermeiro como também responsável pelas orientaçôes acerca dos cuidados que deveriam ser prestados no domicílio ${ }^{12}$. Isso se deve ao fato da falta de uma comunicação mais efetiva entre cuidadores e enfermeiros, que acabam por deixar de exercer sua funçáo de educador com cuidadores e pacientes.

\section{O preparo do cuidador para a alta hospitalar}

As dificuldades enfrentadas pelos cuidadores levam a refletir sobre o grande déficit de orientaçóes às famílias no momento da alta hospitalar ${ }^{18}$. O preparo dos cuidadores nesse momento se torna um instrumento relevante no processo de reabilitaçáo, pois o conhecimento da dimensão e complexidade da nova realidade vivenciada pode contribuir para maior independência, habilitando os cuidadores na tarefa do cuidar ${ }^{13}$. Dessa forma, configura-se como uma importante ferramenta para os cuidados no domicílio ${ }^{10}$. 
Pesquisas sugerem a criaçáo de programas de orientação com informaçóes sobre AVC, suas consequências, adaptaçóes para as atividades da vida diária e apoio continuado ${ }^{19}$. Em um estudo realizado em unidades clínicas de um hospital universitário com vistas a analisar a percepção de cuidadores familiares de idosos com AVC em relação a um programa de alta hospitalar, constatou-se que as orientaçóes após a alta hospitalar fornecidas pelo programa auxiliaram no cuidado no domicilio, os participantes destacaram também habilidades adquiridas e transformação a partir do conhecimento aprendido para realizar o cuidado. A partir das orientaçóes fornecidas pelo programa, os participantes perceberam a importância do resgate da cidadania de seu ente, passando a incentivar a autonomia, o que contribuiu para que o portador de AVC se restabelecesse de suas sequelas e contribuiu para que não evoluísse para um quadro de piora $^{20}$.

Portanto, evidenciou-se a necessidade da criação de estratégias similares com o intuito de amenizar o despreparo do cuidador, contribuindo para uma melhor recuperação, restabelecendo incapacidades e/ou impedindo sua evolução, alcançando melhor qualidade de vida reduzindo o número de hospitalizaçóes desnecessárias ${ }^{20}$.

Programas de alta hospitalar são essenciais para auxiliar na recuperação e reabilitaçáo de pacientes dependentes de cuidados no domicílio, contribuindo assim para minimizar complicaçōes advindas da falta de preparo dos cuidadores domiciliares.

\section{Discussão}

\section{Dificuldades enfrentadas pelos cuidadores familiares}

A carência de informaçóes e de vínculo com os profissionais de saúde acarreta ao cuidador despreparo quanto aos cuidados específicos que precisam ser prestados e como devem ser realizados, dificultando a execuçâo de um cuidado qualificado ${ }^{7}$. Cuidados básicos, como alimentação adequada, distribuições das medicaçōes em horários corretos, forma de administração e mudança de decúbito, são orientações básicas e simples sobre as quais o cuidador deve estar orientado, visto que são fundamentais para manter a condição clínica sob controle ${ }^{7}$.

Portanto, para programar os cuidados a serem prestados, os familiares necessitam conhecer aspectos fundamentais da patologia, suas prováveis consequências e agravos, contribuindo dessa forma para melhoria da qualidade do cuidado a ser executado no domicílio, pois essas informações possibilitam um cuidado mais consciente, responsável e com mais qualidade por parte do cuidador. Isso colabora diretamente para prevenir complicaçóes e proporcionar um bom prognóstico ao paciente ${ }^{7}$.

A sobrecarga dos cuidadores reflete de forma negativa sobre sua própria saúde e a do paciente, pois com o passar do tempo açóes de cuidado como, higiene, conforto, alimentação tornam-se rotineiras, levando a um cansaço físico e mental que compromete a qualidade de vida de ambos ${ }^{21,22}$.

Sendo assim, os cuidadores também devem ser alvos dos profissionais de saúde para que a pessoa dependente de seus cuidados não sofra as consequências dessa sobrecarga e tenha um cuidado de qualidade.

\section{Enfermeiro como educador no sistema de cuidado domiciliar}

A educação em saúde compóe todos os campos de atuação da enfermagem; no caso do AVC, a colaboração com a família é necessária para a execução dos cuidados, mesmo após a alta hospitalar. O familiar executará açóes com o indivíduo incapacitado sem a presença da enfermagem no domicílio. Assim, caso tenha recebido informaçôes sobre a doença e os cuidados necessários, contribuirá para melhoria da qualidade de vida do seu ente $^{23}$.

Dessa forma, o enfermeiro deve utilizar-se de suas habilidades de educador para possibilitar ao cuidador familiar a utilizaçâo, no domicílio, dos conhecimentos adquiridos durante a estadia no hospital. A formação de um elo entre o enfermeiro e a família é enriquecedor e traz benefícios ao paciente, particularmente aos com AVC, que precisam de cuidados especiais de cunho afetivo, emocional e físico ${ }^{24}$.

A equipe de enfermagem no ambiente hospitalar mantém uma relação muito próxima com os pacientes e seus acompanhantes, mais do que a estabelecida com os outros profissionais. Isso ocorre devido à presença da equipe de enfermagem, em tempo integral, a fim de atender as necessidades dos clientes e dos seus familiares ${ }^{23,24}$.

No entanto, existem barreiras que dificultam a inserção da educação em saúde no ambiente hospitalar; essas dificuldades são atribuídas à falta de profissionais, sobrecarga de atividades, desvalorização por parte da instituição hospitalar, pela assistência voltada para o tecnicismo e medicalizaçáo da saúde e pouca valorização por parte dos próprios enfermeiros, que atribuem essa atividade como responsabilidade apenas dos profissionais da atenção básica ${ }^{25}$.

Portanto, torna-se necessária a sensibilização dos profissionais, bem como das instituiçóes públicas ou privadas, para a capacitação de enfermeiros para o exercício da educação em saúde. 


\section{O preparo do cuidador para a alta hospitalar}

As orientaçóes de como o paciente deve ser cuidado em seu domicílio, recebidas na alta hospitalar, proporcionam ao cuidador mais segurança, diminuem a ansiedade e o estresse ${ }^{26}$. Desse modo, uma família que é bem orientada acerca da patologia e suas possíveis complicaçóes sente-se mais segura ao enfrentar as limitaçóes impostas pelo AVC, auxiliando, assim, na recuperação do paciente de forma efetiva. Portanto, a equipe de enfermagem deve preparar a família para aprender a cuidar no domicílio e também a lidar com seus conflitos e medos ${ }^{27}$.

As ações desenvolvidas na alta hospitalar devem ser programadas de acordo com as necessidades de cada paciente, para melhorar a qualidade de vida, prevenir complicações e evitar reincidência de internaçōes ${ }^{26}$. Sendo assim, o preparo para a alta deve acontecer desde o momento da admissão, permitindo o compartilhamento de saberes e contribuindo para o desenvolvimento da autonomia e confiança exigidas para a prática do cuidado no domicílio ${ }^{27}$.

Um dos motivos de reincidência de internaçóes deve-se à falta de preparo adequado do paciente e da família para a alta hospitalar. Dessa forma, torna-se indispensável uma assistência organizada e multidisciplinar com o intuito de minimizar as necessidades e expectativas dos pacientes e familiares, cabendo ao enfermeiro o importante papel de coordenação e planejamento da alta hospitalar ${ }^{28}$.

No entanto, um planejamento não sistematizado influencia de forma negativa a qualidade da assistência, e por isso o enfermeiro deve conhecer de forma integral o paciente, reconhecer suas reais necessidades, capacidades e limitações, estimulando o autocuidado, garantindo a qualidade assistencial no domicílio ${ }^{28}$.

Pode-se constatar a importância do preparo para a alta hospitalar como uma estratégia para o cuidado continuado no domicílio, e mesmo que seja feito o planejamento da alta pelo enfermeiro com o paciente e familiares e que eles encontrem apoio no sistema de saúde, dúvidas podem persistir entre familiares ${ }^{29}$.

Sendo assim, a assistência a esses pacientes deve ser contínua, e não se limita apenas ao ambiente hospitalar. Os enfermeiros e os outros profissionais da saúde devem recomendar aos cuidadores que busquem um serviço de saúde sempre que surgirem dúvidas sobre as situaçóes que envolvem os pacientes dependente dos seus cuidados.

\section{Considerações finais}

O AVC se configura como um grande problema de saúde pública pelo alto índice de morbimortalidade, requerendo, dessa forma, mais atenção dos profissionais de saúde a criaçáo de políticas públicas e programas que visem à melhoria da qualidade de vida da clientela em estudo.

Pôde-se perceber a importância de oferecer uma educaçáo em saúde de qualidade aos pacientes e cuidadores durante o período de internação e pós-alta. Compete ao enfermeiro utilizar-se de suas habilidades de educador como uma estratégia de cuidado permanente ao paciente após a alta hospitalar. Isso deve ser feito por meio da minimização das dúvidas, medos e dificuldades dos cuidadores e da capacitação dos mesmos para enfrentar a nova realidade e proporcionar ao paciente uma melhor qualidade de vida.

Diante do exposto propóem-se a criação de programas de alta hospitalar, capacitaçâo dos profissionais de saúde para atender de forma adequada a problemática do paciente e seu cuidador e a criação de uma cartilha ilustrativa multidisciplinar que envolva vários profissionais da saúde na adequação e reabilitação dos pacientes no âmbito domiciliar, servindo assim como um instrumento de apoio aos profissionais envolvidos na assistência a esses pacientes e a suas famílias, permitindo que atuem como multiplicadores dos conhecimentos adquiridos.

Como limitaçáo observou-se que poucos dados encontram-se disponíveis nas bases de dados LILACS e SciELO sobre a qualificaçáo dos enfermeiros para lidar com cuidadores e pacientes sequelados após um AVC no momento da alta hospitalar. Dessa forma, faz-se necessária a realização de novos estudos que abordem sobre essa temática. Vale ressaltar, no entanto, que a limitação apresentada não diminui a relevância do estudo realizado.

\section{Referências}

1. Smeltzer SC, Bare BG. Brunner, Suddarth. Tratado de enfermagem médico-cirúrgica. Rio de Janeiro: Guanabara Koogan; 2011. 1899-914.

2. Brasil. Ministério da Saúde. Acidente vascular cerebral: uma das principais causas de mortes no mundo, doença pode ser prevenida com hábitos saudáveis de vida [Internet]. Brasília: Ministério da Saúde; 2012. Disponível em: <http://www. brasil.gov.br/saude/2012/04/acidente-vascular-cerebral-avc>. [Acesso em 18 mar. 2014].

3. Brasil. Ministério da Saúde. Em 10 anos, país reduz em $32 \%$ mortes por AVC [Internet]. Brasília: Ministério da Saúde; 2012. Disponível em: <http://www.brasil.gov.br/ saude/2012/10/em-10-anos-pais-reduz-em-32-mortes-por-avc>. [Acesso em 21 fev. 2014].

4. Falção IV, Carvalho EMF, Barreto KML, Lessa FJD, Leite VMM. Acidente vascular cerebral precoce: implicaçóes para adultos em idade produtiva atendidos pelo Sistema Único de Saúde. Rev Bras Saúde Mater Infant. 2004;4(1):95-102. 
5. Arantes NF, Vaz DV, Mancini MC, Pereira MSDC, Pinto FP, Pinto TPS. Efeitos da estimulação elétrica funcional nos músculos do punho e dedos em indivíduos hemiparéticos: uma revisão sistemática da literatura. Rev Bras Fisioter. 2007;11(6):419-27

6. Paiva RS. Ressignificando a alta hospitalar e percebendo-se como o cuidador familiar: um estudo de enfermagem [dissertação]. Rio de Janeiro (RJ): Universidade Federal do Rio de Janeiro, 2011.

7. Teston EF, Oliveira AP, Marcon SS. Necessidades de educação em saúde experienciadas por cuidadores de indivíduos dependentes de cuidado. Rev Enferm UERJ. 2012;20(6):720-5.

8. Chagas NR, Monteiro ARM. Educação em saúde e família: o cuidado ao paciente, vítima de acidente vascular cerebral. Acta Sci Health Sci. 2004;26(1):193-204.

9. Andrade OG, Rodrigues RAP. Abordagem holística do sistema de cuidado familiar do idoso com acidente vascular cerebral. Ciên Cuid Saúde. 2002;1(1):185-91.

10. Perlini NMOG, Faro ACM. Cuidar de pessoa incapacitada por acidente vascular cerebral no domicílio: o fazer do cuidador familiar. Rev Esc Enferm USP. 2005;3(9):154-63.

11. Andrade LM, Costa MFM, Caetano JÁ, Soares E, Bezerra EP. A problemática do cuidador familiar do portador de acidente vascular cerebral. Rev Esc Enferm USP. 2009;1(1):37-43.

12. Souza CB, Abreu RNDC, Brit EM, Moreira TMM, Silva LMS, Vasconcelos SMM. O cuidado domiciliar de idosos acometidos por acidente vascular cerebral: cuidadores familiares. Rev Enferm UERJ. 2009 jan-mar;1(17):41-5.

13. Garanhani MR, Alves JF, Fugisawa DS, Garanhani ML. Adaptação da pessoa após acidente vascular encefálico e seu cuidador: ambiente domiciliar, cadeira de rodas e de banho. Acta Fisiatr. 2010;4(17):164-8.

14. Pereira RA, Santos EB, Fhon JRS, Marques S, Rodrigues RAP. Sobrecarga dos cuidadores de idosos com acidente vascular cerebral. Rev Esc Enferm USP. 2013;1[47]:185-92

15. Bocchi SCM, Angelo M. Interação cuidador familiar-pessoa com AVC: autonomia compartilhada. Ciên Saúde Colet. 2005;3(10):729-38.

16. Bocchi SCM. O papel do enfermeiro como educador junto a cuidadores familiares de pessoas com AVC. Rev Bras Enferm. 2004;5(57):569-73.

17. Torres IEF, Prieto AM, Lián AH. Calidad de vida de cuidadores de adultos com accidente cerebrovascular. Av Enferm. 2010;XXVIII(90).
18. Machado ALG, Jorge MSB, Freitas CHA. A vivência do cuidador familiar de vítima de acidente vascular encefálico: uma abordagem interacionista. Rev Bras Enferm. 2009;2(62):246-51.

19. Garanhani MR, Ferreira AMDM, Silva CK, Laskovsk L, Moreira MD, Garanhani ML. A experiência de pacientes e cuidadores após acidente vascular encefálicos: uma revisão narrativa da literatura. Ciên Cuid Saúde. 2009; 8(2):242-9.

20. Cesar AM, Santos BRL. Percepção de cuidadores familiares sobre um programa de alta hospitalar. Rev Bras Enferm. 2005;6(58):647-52.

21. Faller JW, Barreto MS, Ganassin GS, Marcon SS. Sobrecarga e mudanças no cotidiano de cuidadores familiares de paciente com doença crônica. Ciên Cuid Saúde. 2012;11(1):181-9.

22. Loureiro LSN, Fernandes MGM, Nóbrega MML, Rodrigues RAP. Sobrecarga em cuidadores familiares de idosos: associação com características do idoso e demanda de cuidado. Rev Bras Enferm. 2014;67(2):227-37.

23. Rodrigues LS, Alencar AMPG, Rocha EG. Paciente com acidente vascular encefálico e a rede de apoio familiar. Rev Bras Enferm. 2009;62(2):272-7.

24. Santos AG, Costa NAM. Atendimento da equipe de saúde a paciente vítimas de acidente vascular cerebral. Rev Rede Cuid Saúde. 2012;6(2):1-13.

25. Figueira AB, Amestoy SC, Cecagno D, Tristão FS, Trindade LL, Correa VA. Visão do enfermeiro frente à prática da educaçẫo em saúde no ambiente hospitalar. Cogitare Enferm. 2013;18(2):310-6.

26. Bernardino E, Segui MLH, Lemos MB, Peres AM. Enfermeira de ligação: uma estratégia de integração em rede. Rev Bras Enferm. 2010;63(3):459-63.

27. Paiva RS, Valadares GV. Vivenciando o conjunto de circunstâncias que influenciam na significação da alta hospitalar: estudo de enfermagem. Esc Anna Nery. 2013; 17(2):249-55.

28. Souza PMBB, Queluci GC. Considerações sobre os cuidados de enfermagem aos pacientes na alta hospitalar: revisão integrativa. Rev Enferm UFPE. 2013;7(esp):6238-44.

29. Delatorre PG, Sá SPC, Valente GSC, Silvino ZR. Planejamento para a alta hospitalar como estratégia de cuidado de enfermagem: revisão integrativa. Rev Enferm UFPE. 2013;7(esp):7151-9. 